\title{
LA TUTELA Y REFORMA EN SALUD: DESENCANTO DE UN JUEZ SOÑADOR*
}

\author{
THE WRIT AND HEALTH REFORM: \\ DISILLUSIONMENT OF A DREAMER JUDGE
}

\author{
Carlos Mario Molina Betancur**
}

Fecha de recepción: 19 de junio del 2013 Fecha de aceptación: 21 de julio del 2013

\section{Para citar este artículo / To cite this article}

\author{
Molina Betancur, Carlos Mario, La tutela y reforma en salud: desencanto \\ de un juez soñador, 127 Vniversitas, 157-188 (2013) \\ doi:10.1144/Javeriana.VJ127.trsd
}

* Artículo producto de la investigación "El impacto de la tutela en salud", terminada en el Centro de Investigaciones Jurídicas de la Universidad de Medellín en el 2010.

** Abogado de la Universidad de Medellín, Doctor en Derecho Público de la Universidad Panthéon-Assas-Paris II. Director del Doctorado en Derecho de la Universidad de Medellín, Director de la Maestría en Derecho de la Universidad de Medellín, Director del Grupo de Investigaciones Jurídicas de la Universidad de Medellín, Comisionado de la Sala de Maestrías y Doctorados del Ministerio de Educación, Director de la Asociación Colombiana de Facultades de Derecho, Acofade, Director del Centro de Pensamiento D y L Derechos y Libertades, Conjuez en el Consejo Superior de la Judicatura. Correo electrónico: cmolina@ udem.edu.co 


\section{RESUMEN}

En el grupo de los derechos sociales, económicos y culturales, el avance normativo colombiano en materia de salud ha sido de los más destacados en el mundo en las últimas décadas. La Corte Constitucional, obviando la naturaleza constitucional de estos derechos, ha reconocido en ciertos casos la prestación de salud como un derecho subjetivo, protegido como derecho fundamental en instancia de tutela. Para el gobierno y un sector de la doctrina, esto ha generado problemas de financiación y desorden en la estructura del Sistema de Seguridad Social, pero para otro sector de la doctrina esto no es sino una consecuencia lógica del nuevo constitucionalismo. A pesar de todos estos esfuerzos estructurales y avances jurisprudenciales, el Estado no ha podido consolidar la protección que garantice el acceso y la calidad en los servicios de salud, además la amplia protección jurisprudencial sigue siendo frágil e incierta.

Palabras clave autor: Tutela en salud, reforma en salud, juez constitucional.

Palabras clave descriptor: Acción de tutela, sistema general de seguridad social en salud, Reforma de la Atención de Salud, Colombia. 


\section{ABSTRACT}

In the group of social, economic and cultural rights, the Colombian regulatory progress in health has been the most prominent in the world in recent decades. The Constitutional Court, bypassing the constitutional nature of these rights, recognized in some cases the provision of health as an individual right, protected as a fundamental right for writ instance. For the government and a sector of the doctrine, this has generated funding problems and disorder in the structure of the social security system, but for the other part of this doctrine is but a logical consequence of the new constitutionalism. Despite all these efforts and structural developments in jurisprudence, the state has failed to consolidate protection to ensure access and quality of health services, besides comprehensive protection jurisprudence remains fragile and uncertain.

Keywords author: Health writ, health reform, constitutional judge.

Keywords plus: Writ of protection, general social security health, Health Care Reform, Colombia.

\section{SUMARIO}

INTRODUCCIÓN.- I. LA JUSTICIABILIDAD DEL DERECHO A LA SALUD.- A. Un reconocimiento mundial del derecho a la salud como derecho social.- B. Un reconocimiento especial de justiciabilidad en Colombia.- II. LA FRAGILIDAD DE JUSTICIABILIDAD DEL DERECHO A LA SALUD EN COLOMBIA.- $A$. Discusiones acerca de la justiciablidad del derecho a la salud.- B. Límites a la justiciabilidad del derecho a la salud.- CONCLUSIÓN. 


\section{INTRODUCCIóN}

La acción de tutela en materia de salud se ha convertido en una verdadera "revolución pacífica" en nuestro país". Pero como toda revolución, ha sido necesario un tiempo prudente para asimilar el cambio en materia de protección de derechos, además de que ni el gobierno ni la sociedad estaban preparados para afrontar el nuevo Estado social de derecho que proclamó la Constitución de $1991^{2}$. Fue precisamente esta Constitución la que cambió los esquemas en materia de protección de derechos constitucionales de carácter social, en especial el derecho a la salud ${ }^{3}$. Posteriormente, inspirada en el modelo chileno, primer sistema de salud en Latinoamérica en privatizar el sector salud, fue la Ley 100 de 1993 la que fundamentó la creación del Sistema General de Seguridad Social en Salud - SGSSS en Colombia.

Nuestro modelo mixto de competencia regulada, el cual garantizaría mayor cobertura y eliminación del monopolio del Estado, conservó una parte de la antigua estructura del seguro social y privatizó la otra parte del sistema, incorporando paulatinamente la participación de agentes privados en el aseguramiento y la prestación de servicios de salud, con el compromiso de atender una parte de la población no asegurada laboralmente que estaría asistiendo a los establecimientos privados en calidad de subvencionados.

De esta forma, el Estado liberó el mercado para conseguir los mejores resultados sociales, no sin antes advertir que dicha liberación quedaría bajo la regulación, dirección, vigilancia y control estatal. Es decir, que el servicio a la salud adquirió una doble naturaleza ${ }^{4}$. Tanto de servicio público como de derecho social; lo que requiere de antemano de un desarrollo legal que los establezca, un árbitro imparcial que los vigile y una estructura financiera que los garantice ${ }^{5}$.

Durante los primeros años de aplicación de la jurisprudencia de la Corte Constitucional (1992-2000), que conecta el derecho a

1 Contraloría General de la República, El sistema nacional de salud diez años después de la Ley 100, Revista Economía Colombiana, No. 303 (2003).

2 O. Cortés Hernández, Derecho a la Seguridad Social, $2^{\text {a }}$ ed., Librería ediciones del profesional Ltda., Bogotá, 3 (2006) .

3 M. Arbeláez Rudas, Derecho a la salud en Colombia, el acceso a los servicios del sistema general de seguridad social en salud, IDEP-CINEP-Colciencias, Bogotá (2006).

4 Corte Constitucional. Sentencia T-406. 1993. M.P. Alejandro Martínez Caballero.

5 Corte Constitucional. Sentencia T-042. 1995. M.P. Carlos Gaviria Díaz. 
la salud de manera fundamental con el derecho a la vida, poco fue el impacto que se presentó en el sector salud. Sin embargo, se le reconoce a la Corte la "osadía" de haber consagrado dicho derecho como fundamental, aun de manera tímida al momento de autorizar los tratamientos médicos más importantes, que no estuvieran incluidos en los planes obligatorios de salud o en el régimen subvencionado ${ }^{6}$.

A partir de la segunda generación jurisprudencial (2000-2008), comienza la Corte Constitucional a precisar mejor la protección constitucional del derecho a la salud como un derecho social fundamental: se cambian muchos aspectos jurisprudenciales, se amplía considerablemente la cobertura y se dejan listas las bases para implementar una verdadera política jurisprudencial en materia de derechos económicos, sociales y culturales?.

El impacto jurisprudencial de dicha protección sobre el sistema de salud comienza a verse reflejado en esta época por el alto volumen de tutelas que llegan a todos los juzgados del país. Con esto parece ponerse en entredicho la sostenibilidad financiera de dicha protección, y por ello comienzan a conocerse serios requerimientos importantes por parte de uno de los organismos de control más representativos del país 8 .

A estas voces precedían otras ${ }^{9}$ que habían hecho análisis profundos desde el 2003 sobre el comportamiento de las tutelas en el sector salud, revelando el impresionante desbordamiento de las demandas de tutela en Colombia y su preocupante situación por su desacato en la administración pública. A pesar de los esfuerzos del gobierno por mejorar la situación, con la expedición de la Ley 1122 de 2007, los estudios arrojan resultados inesperados sobre la vulneración casi general del derecho a la salud: más de 60.000 tutelas anuales solicitaban la protección fundamental de la salud de los colombianos por el desconocimiento de las autoridades encargadas de protegerlo y garantizarlo. La cuestión comienza a ser alarmante.

6 Defensoría del Pueblo. El derecho a la salud en la Constitución, la jurisprudencia y los mecanismos internacionales, Defensoría del Pueblo, Bogotá (2004).

7 N. Paredes, El derecho a la salud: su situación en Colombia, CINEP-GTz, Bogotá (2003).

8 Procuraduría General de la Nación, Informe en Salud, Bogotá (2008)

9 Defensoría del Pueblo, El derecho a la salud en la Constitución, la jurisprudencia y los mecanismos internacionales, Defensoría del Pueblo Bogotá, (2004). 
En medio de estas denuncias, interviene la Sentencia T-760 ${ }^{10}$, que marca una etapa de consolidación de la revolucionaria jurisprudencia inicial en materia de protección al derecho a la salud en Colombia. A partir de allí, con un activismo judicial más pronunciado, comienza una tercera generación jurisprudencial de protección fundamental del derecho a la salud, con un impacto considerable en las finanzas del Estado.

Pronto el gobierno interviene el sistema con varios decretos de emergencia social que pretenden atacar el problema funcional que comienza a tener falencias estructurales. Para la academia, el remedio podría llegar a ser más grave que la enfermedad, y por ello se pronuncia rápidamente y exige del gobierno un cambio considerable en la estructura del sistema de salud, emplazando al gobierno para reformar el sistema de financiamiento de forma global ${ }^{11}$.

Con esto se empieza a decir, como lo hacen frecuentemente algunos columnistas de la prensa en el país ${ }^{12} \mathrm{o}$ algunos investigadores que han tratado el tema ${ }^{13}$, que "la salud de los colombianos estaba enferma": el aumento de las demandas de protección por tutela, de un lado, parecían estar desfondando el presupuesto estatal, instaurado para su financiamiento y subvención, y de otro lado, asfixiando el trabajo de los jueces del país.

El problema es que el panorama tiende a agravarse, con la autorización por parte de la Corte Constitucional del derecho parcial al aborto $^{14}$; la preocupante situación del virus gripal en el mundo; los problemas de alcoholismo juvenil, que para algunos comienza a los 10 años; el alto porcentaje de adicción de la población a las drogas ilícitas, que para otros comienza a los 12 años; los altos índices de aborto en las jóvenes de entre 15 y 19 años; los brotes generales de paludismo; del dengue endémico; de tuberculosis y hasta de rabia y gripe AH1N1.

10 Corte Constitucional. Sentencia T-760 de 2008. M.P. Manuel José Cepeda Espinosa.

11 N. Ardón \& A. Centeno, Voces críticas desde la academia sobre La emergencia social en salud. Cuadernos del doctorado, No.13, Universidad Nacional de Colombia, Bogotá (2006). Disponible en: http://web.saludsantander.gov.co/index.php?option=com_docman\&task=doc_ download\&gid=263\&Itemid=7

12 F. Londoño Hoyos, "Extraña Paradoja", El Tiempo, 11 de febrero del 2011.

13 C. Molina, Reflexiones acerca del activismo judicial en materia de derechos sociales en Colombia: el caso del derecho a la salud, Revista Elementos de Juicio, No. 13, 91-113. ISBN. 978-958-98201$2-4$.

14 C. Molina. El derecho al aborto: segunda parte, la despenalización parcial, Universidad de Medellín. ISBN.978-958-8348-97-1. 
Sin embargo, la inversión en salud seguía siendo muy baja: esta equivalía en 2008 al 8\% del PIB, unos $\$ 18$ billones de pesos; para el 2010 llegaba al 10\%, lo que equivalía a unos $\$ 23$ billones y representaba muy poco para el tamaño del sistema: con un financiamiento cada vez más próximo de la cobertura total, pero más alejados de la calidad mínima. Esta divergencia entre calidad y cantidad genera paulatinamente una polémica importante en nuestro país, acostumbrado a ver morir a muchos de sus ciudadanos en las puertas de los hospitales por falta de capacidad financiera para subvencionar a los más desfavorecidos económicamente.

Hoy el panorama ha cambiado considerablemente: el gobierno se ha personalizado del problema, creando un Sistema General de Seguridad Integral en Salud, el cual, así tenga algunas fallas de concepción y no funcione por el momento, es un gran paso en el desarrollo social de nuestra nación (I). Sin embargo, la nueva reforma quiere controlar de cierta forma la excesiva justiciabilidad del derecho a la salud, lo que limitaría en cierta forma su grado de protección (II).

\section{LA JUSTICIABILIDAD DEL DERECHO A LA SALUD}

El reconocimiento de protección judicial en salud es reconocido en el mundo entero, con especial énfasis en Colombia.

\section{A. Un reconocimiento mundial del derecho a la salud como derecho social}

Los orígenes de la seguridad social en el mundo se encuentran en Inglaterra a mediados del siglo XVII ${ }^{15}$. La primera legislación en la materia data de 1601 en Inglaterra con el primer impuesto obligatorio para asistir a los más necesitados. Años más tarde, Francia expide una reglamentación del mismo estilo, denominada "Reglamento Colbert", en honor al más célebre ministro de finanzas de Luis XIV. Dicho reglamento estableció un descuento automático sobre el salario de los trabajadores del reino, sobre todo de aquellos

15 O. Cortés Hernández, Derecho a la Seguridad Social, $2^{\text {a }}$ ed., Librería ediciones del profesional Ltda., Bogotá, 3 (2006). 
que ejercían profesiones peligrosas o de riesgo: obreros de puentes y calzadas, cazadores, conductores, jardineros, mensajeros, cobradores de impuestos, leñadores y cocineros, para garantizar sus gastos de atención en caso de accidente o para cubrir su hospitalización.

Con el advenimiento revolucionario, los derechos individuales hacen su aparición en Europa, estableciendo leyes parlamentarias que promulgan por primera vez en 1793 reglamentaciones más completas en materia de salud y pensiones. Un ejemplo de esto lo constituye el primer proyecto de pensiones que garantiza la atención de supervivencia de las personas sin capacidad de pago como aquellas lesionadas o mutiladas sin acceso al empleo.

Mucho más tarde en Alemania, a finales del siglo XIX, el canciller Bismarck adoptó el concepto de Versicherung, el cual se adoptó como un contrato de seguro que contiene derechos y obligaciones recíprocas entre el empleador y el empleado, encaminadas a garantizar la protección obligatoria del trabajador y su núcleo familiar ${ }^{16}$. Con esto se acuña la expresión, hoy válida en el mundo entero, del "seguro social" como sistema solidario y obligatorio de aportes, entre el empleador y el empleado, con garantía de sostenibilidad de parte del Estado, el cual se extiende no solamente a los riesgos de accidentes y muerte sino también al de enfermedades y pensiones, en Inglaterra en 1897 y en Francia en 1898.

Hacia los años treinta, los movimientos obreros ingleses realizan reivindicaciones más generales de protección social abriendo paso a la idea de un Estado nuevo, denominado "Estado social de derecho"17, término jurídico acuñado después políticamente por el político Louis Blanc en Francia ${ }^{18}$ como el "Estado de derecho democrático y social". Esta protección encuentra su consolidación más moderna en el concepto de Estado social de derecho en la época de la posguerra de los años cincuenta en Europa ${ }^{19}$, especialmente en aquellos países que quedaron devastados por un enfrentamiento bélico de proporciones mundiales: Francia, Inglaterra y Alemania.

16 N. Paredes, El derecho a la salud: su situación en Colombia, CINEP-GTZ, Bogotá (2003).

17 H. Heller, Teoría del Estado, Fondo de Cultura Económica, México D.F. (1983).

18 W. Abendroth, El Estado de derecho democrático y social, en: vvvaA, El Estado social, Centro de Estudios Constitucionales, Madrid (1986).

19 J. Pérez Royo, El Estado social de derecho, en: Enciclopedia Jurídica Básica, Vol. III, Civitas, Madrid, 2924 (1989). 
En América, el sistema llega solamente hasta 1924 en Chile, en 1935 a Estados Unidos, en 1936 al Perú. En Colombia, el sistema solamente verá la luz en 1936 bajo la presidencia de Alfonso López Pumarejo, con la influencia de Roosevelt en el gobierno del New Deal latinoamericano.

Lo anterior permite un desarrollo importante en materia de asistencia social en el mundo, en donde el Estado asume su rol de asistente social y lo integra dentro de su ordenamiento jurídico como un deber frente a la Declaración de Derechos aceptados progresivamente por todos los Estados liberales. De esta forma, la noción de servicio público se abre paso en Francia, en donde se cubren las necesidades básicas de los ciudadanos en materia de salubridad, seguridad y salud. Los hospitales públicos comienzan a florecer y con ellos una idea de Estado bienestar a quien le corresponde velar por la salud de todos.

A pesar de estos avances, estos servicios nunca fueron considerados como un derecho sino como una competencia del Estado, la cual debía administrar lo mejor posible de acuerdo a sus posibilidades económicas. La salud aparece solamente como un derecho en 1946 en el Preámbulo de la Constitución francesa, según la cual, " $L a$ Nación asegura a todos, principalmente a los niños, a las madres y a los trabajadores, la protección de la salud”. En Portugal se establece en el artículo 64.1 que "Todos tendrán derecho a la protección de la salud y el deber de defenderla y promoverla". De igual manera en España, el artículo 43 de la Constitución de 1978 establece que "se reconoce a la protección de la salud”. En este sentido se ha pronunciado la legislación mundial:

- Constitución de la Organización mundial de la Salud de 1946.

- Declaración Universal de Derechos Humanos de 1948.

- Carta Social Europea de 1961.

- Pacto Internacional de Derechos Económicos, Sociales y Culturales de 1966.

- Convención Americana de Derechos Humanos de 1969.

- Carta Africana de Derechos Humanos y de los Pueblos de 1981.

- $\quad$ Acta Única Europea de 1987. 
- Protocolo Adicional de la Convención Americana sobre Derechos Humanos en materia de Derechos Económicos, Sociales y Culturales de 1988.

- Declaración de los Derechos y Libertades Fundamentales de 1989.

- Carta Comunitaria de los Derechos Sociales Fundamentales de los Trabajadores de 1989.

Así, desde la época de postguerra, la Organización Mundial de la Salud define a la salud no solamente como la ausencia de afecciones o de enfermedades sino como un estado completo de bienestar físico, mental y social. Su constitución fue adoptada por la Conferencia Sanitaria Internacional, celebrada en Nueva York del 19 de junio al 22 de julio de 1946, firmada el 22 de julio de 1946 por los representantes de 61 Estados (Off. Rec. Wld Hlth Org.; Actes off. Org. mond. Santé, 2, 100), y entró en vigor el 7 de abril de 1948. Las reformas adoptadas por la $26^{\text {a }}$, la $29^{\mathrm{a}}$, la $39^{\mathrm{a}}$ y la $51^{\mathrm{a}}$ Asambleas Mundiales de la Salud (resoluciones WHA26.37, WHA29.38, WHA39.6 y WHA51.23), que entraron en vigor el 3 de febrero de 1977, el 20 de enero de 1984, el 11 de julio de 1994 y el 15 de septiembre de 2005, respectivamente, se han incorporado al presente texto. En el mismo sentido se lee el Convenio 169 de la Organización Internacional del Trabajo.

Por consiguiente, el derecho a la salud comprende: la atención, tanto individual como social, de las patologías, de los tratamientos, así como de las actividades de prevención, promoción y protección. Ello definiría entonces jurídicamente el derecho a la salud como la garantía que debe tener un ser humano de acceder a ciertas prestaciones mínimas que le ayuden a mantener o a recuperar su salud física y mental ${ }^{20}$.

Lo que buscaba dicha organización era la garantía política para que las personas pudieran llevar una vida sana dentro de una sociedad más justa. El concepto de salud, entendido desde una perspectiva social amplia ${ }^{21}$, incluía entonces aspectos importantes y complementarios tales como: una adecuada alimentación, unas

20 oms - Unicef, Salud para todos en el siglo XXI, en: Declaración de Alma Alta, Ginebra (1999).

21 M. Restrepo, Elementos para una doctrina del derecho a la salud en Colombia, en: El ejercicio del derecho a la salud en Colombia, Defensoría del Pueblo, Bogotá, 60 (2002). 
condiciones mínimas de salubridad, el fácil acceso al agua potable, las condiciones sanitarias adecuadas, vivienda digna, vida sexual y reproductiva sana, así como un medioambiente adecuado.

Con esto, el reconocimiento constitucional del derecho a la salud no es una declaración imperativa sobre el estado natural de salud que tienen los seres humanos, sino una norma amplia no categórica sobre el derecho al fácil acceso de las prestaciones que el Estado imparte mediante políticas públicas de protección para el mantenimiento de ese estado natural, en este caso el derecho a la protección de ese derecho como rol principal del Estado social de derecho ${ }^{22}$.

Es por esta razón que el derecho a la salud está comúnmente asociado al derecho a la atención y asistencia sanitaria por parte de un especialista en salud. Así lo consagraron las primeras constituciones: en Francia, en 1946 en el Preámbulo de la Constitución, se dice: "La Nación asegura a todos, principalmente a los niños, a las madres y a los trabajadores, la protección de la salud”. Portugal en el artículo 64.1 "Todos tendrán derecho a la protección de la salud y el deber de defenderla y promoverla". En España, el artículo 43 de la Constitución de 1978 establece "se reconoce a la protección de la salud".

Dicho derecho incluye no solamente el acceso curativo sino también el preventivo y el pos-tratamiento, lo que dificulta enormemente una garantía universal y gratuita de todo el servicio aun en los países desarrollados, con mayor razón entonces en los países subdesarrollados donde no se cuenta con los medios necesarios para hacerlo.

Lo anterior reconforta la tesis de la naturaleza programática del derecho a la salud como derecho social, en donde se deben establecer una serie de políticas públicas dirigidas a la protección de dicho derecho mediante una adecuada prestación del servicio. La mayor parte de las Constituciones del mundo consagran esta protección dentro de sus cláusulas de derechos económicos, sociales y culturales, en donde los derechos son identificados como normas programáticas que brindan principios ordenadores de las políticas sociales.

22 H. Hart, El concepto de derecho, Abeledo Perrot, Buenos Aires (1990). 
En este sentido, la doctrina mayoritaria española, al analizar el enunciado del artículo 53.3 de la Constitución española, considera que el derecho a la salud, como derecho perteneciente a la categoría de derechos económicos, sociales y culturales, no es un verdadero derecho ${ }^{23}$. Este derecho es de un enunciado programático, que obliga al Estado a expedir una legislación de desarrollo que reglamente adecuadamente las provisiones presupuestales del gobierno de la asistencia y protección del derecho a la salud ${ }^{24}$, por lo tanto, está por fuera de la protección judicial que garantiza el amparo constitucional ${ }^{25}$.

En la misma dirección va la doctrina italiana ${ }^{26}$, que al analizar la naturaleza jurídica del artículo 32 de la Constitución establece que el derecho a la salud, por su naturaleza, no se podría erigir en derecho subjetivo sino como cláusula normativa que establece un principio rector de mandato constitucional para los poderes públicos con el fin de condicionar su accionar en las políticas públicas referidas a la salud. Dichas cláusulas no son derechos atribuidos directamente al ciudadano ni son directamente vinculantes para las entidades públicas, por lo cual su justiciabilidad por incumplimiento no está garantizada. Para la doctrina extranjera, toda exigibilidad directa debe estar autorizada y reglamentada adecuadamente por el legislador.

De igual forma ocurre en América Latina, en donde todas las Constituciones, entre ellas la colombiana (artículo 44 de la Constitución de 1991), ubican el derecho a la salud dentro de la categoría de derechos sociales y su garantía va más dirigida hacia la protección del derecho por medio de políticas públicas que a la exigibilidad judicial inmediata del mismo ${ }^{27}$. En Chile, por ejemplo, el artículo 19 de la Constitución de 1980 asegura el derecho a la protección de la salud; en Brasil, el artículo 196 de la Constitución de 1998 establece que la salud es un derecho y un deber del Estado, garantizado me-

23 J. Fernández, El servicio público de la sanidad: el marco constitucional, Civitas, Madrid, 82 (1984).

24 E. Cobrero, Reflexión general sobre la eficacia normativa de los principios constitucionales rectores de la política social y económica del Estado, Revista Vasca de Administración Pública, No. 19, 221 (1987).

25 Tribunal constitucional español. Autos ATC 519 y 564 (1984).

26 M. Mazziotti, Diritti sociali, Milán, D.T.XII, 800 (1964).

27 C. Herrera, Sur le statut des droits sociaux. La constitutionnalisation du social, 16 RUDH, No. $1-4,33$ (2004). 
diante políticas sociales y económicas que tiendan a la reducción del riesgo de enfermedad. Así también se expresa la Constitución de Ecuador de 1998, en su artículo 42, el cual dispone que el Estado garantiza el derecho a la salud por medio del desarrollo de la seguridad alimentaria, la provisión de agua potable y saneamiento básico, así como a través del fomento del ambiente saludable. En el Perú, el artículo 7 del capítulo segundo de la Constitución de 1993 garantiza el derecho a la protección de la salud, y en México, el artículo 4 de la Constitución de 1917 establece que toda persona tiene derecho a la protección de la salud ${ }^{28}$.

Dichas políticas se desarrollan, generalmente, por medio de leyes que obligan a los gobiernos a implementar ciertos mecanismos de protección de acuerdo a sus capacidades económicas anteriormente presupuestadas, por lo cual su total cubrimiento no podría ser exigido directamente por los ciudadanos.

Si esta es la generalidad de los sistemas de protección del derecho a la salud, esto no obsta para que algunos Estados como el colombiano garanticen jurisprudencialmente su prestación.

\section{B. Un reconocimiento especial de justiciabilidad en Colombia}

En Colombia, después de la Constitución de 1991, es la Corte Constitucional y no el texto constitucional quien de forma especial ha sentado los parámetros que han permitido desarrollar muchos de los derechos consagrados en la Carta, especialmente, el derecho a la salud. La naturaleza jurídica del derecho a la salud está establecida en la Constitución Política, artículo 49, como un servicio social, perteneciente al grupo de derechos de segunda generación o de los llamados derechos de atención, programáticos, denominados económicos, sociales y culturales.

Dicha categoría de derechos constitucionales se encuentra caracterizada por el hecho de que todos estos derechos no se adquieren por el solo hecho de ser persona, sino porque dichas personas se encuentran incluidas dentro de una sociedad; es así como el derecho a la salud es entendido en primera medida como un derecho social

28 O. Correas, Acerca de los derechos humanos, Ediciones Coyoacán, México (2003). 
independiente del hecho de ser persona, y que a diferencia de los derechos fundamentales, tiene que estar desarrollado previamente por la ley para ser aplicado ${ }^{29}$.

La Constitución de 1991 establece que el servicio de salud presenta ciertas características que lo convierten en esencial: es irrenunciable, universal y prioritario. Con estas características, el servicio de salud en Colombia es dirigido, coordinado, controlado y vigilado por el Estado; dicho servicio, así se preste por particulares y de manera descentralizada, sigue siendo un servicio al cual se le impone una atención integral, eficiente y solidaria ${ }^{30}$.

De esta manera, el Estado se convierte en el director planificador de la economía social, según el artículo 334, para buscar de forma prioritaria el bienestar de los asociados, en procura de una mejor calidad de vida, una distribución equitativa de los recursos y un mejor desarrollo económico y cultural. Este artículo le brinda al Estado la competencia de intervenir en las políticas de empleo con el fin de asegurar a todas las personas, en prioridad a los de menores ingresos, un fácil acceso a los bienes y servicios básicos. Así, el artículo 336 establece el bienestar general, el mejoramiento de la calidad de vida de la población y la obligación de satisfacer las necesidades básicas insatisfechas en el campo de la salud. Por ello, el artículo 339, establece que el Gobierno Nacional debe fijar las metas para la orientación de la política económica, social y ambiental del país.

Estas competencias económicas, enmarcadas dentro de parámetros sociales, son una gran responsabilidad que le impone al Estado el Constituyente de 1991. Para facilitarle la tarea, el Constituyente dota al Estado de herramientas importantes, como la que le brinda en artículo 340 al Consejo Nacional de Planeación, con representación de las entidades territoriales y de los diversos sectores económicos y sociales del país. Desde allí se establecen políticas generales y se acuerda el Plan Nacional de Desarrollo.

Además, no solamente los gastos están ahora controlados como límites al ejercicio regaliano que ha tenido el gobierno para desti-

29 O. Cetina Vargas, Derecho integral de seguridad social, Universidad Externado de Colombia, Bogotá (1986).

30 A. Rodríguez Castillo, La garantía de la salud como derecho integral en Colombia. Diálogo pendiente, Ed. Planeta Paz, Bogotá (2005). 
nar los recursos del Estado, también los recaudos están, según el artículo 363, limitados por los principios de equidad, eficiencia y progresividad. Ello que implica un tratamiento preferencial para los sectores más desfavorecidos de la sociedad y un manejo eficiente con calidad progresiva de todos los recaudos que imponga el Gobierno Nacional.

Con esto, y a falta de definición constitucional, la justicia colombiana ha definido el derecho a la salud: "como la facultad orgánica funcional tanto física como mental y de restablecer cuando se presente una perturbación en su estabilidad orgánica y funcional, facultad que tiene todo ser humano por el solo hecho de existir"31.

Lo anterior se ha fundamentado categóricamente desde 1994 como un derecho fundamental, el cual se expresa como "El derecho a la salud es un derecho fundamental, derivado del derecho a la vida que tiene toda persona humana desde la concepción hasta la muerte, derecho que implica conservar la plenitud de sus facultades físicas, mentales y espirituales y poner todos los medios ordinarios para la prevención de enfermedades así para la recuperación [...]"32.

En tal sentido, los mecanismos de protección de estos derechos, como la acción de tutela, se podrían ejercer en todos los casos en que se vulnere o se ponga en peligro su ejercicio. Dicho criterio a lo largo de los años ha sido modulado y la Corte ha venido reconociendo a la salud como un servicio público de amplia configuración legal y no de forma general como un derecho fundamental en sí mismo, en la medida en que la Constitución (art. 49 "[...] corresponde al Estado organizar, dirigir y reglamentar la prestación de servicios de salud [...]") difiere en la ley el desarrollo del derecho a la salud, tendiendo como punto de partida el sistema que organice para cumplirlo.

Es válido mencionar en este punto que la libertad configurativa del legislador en materia de derecho a la salud, como perteneciente a la regulación laboral y de seguridad social, se encuentra limitada por la calidad de derecho prestacional (económicos, sociales y culturales), que aunque no siempre puede considerarse como fundamental, salvo por conexidad con un derecho fundamental o cuando se trata de los niños, encuentra cierta protección en lo que se ha denominado "mandato de progresividad", entendido como

31 Corte Constitucional. Sentencia SU-508 de 2001.

32 Corte Constitucional. Sentencia T-447 del 13 de octubre de 1994. M.P. Vladimiro Naranjo. 
la obligación del legislador de respetar los grados de protección alcanzados por los trabajadores en este caso, so pena de inconstitucionalidad, limitándose así la facultad configurativa del ente legislativo.

Dicho mandato de progresividad se traduce entonces en la restricción del legislador para configurar normas jurídicas que retrocedan los derechos y las garantías ya alcanzados por los trabajadores y afiliados al sistema de salud; no obstante, dicha prohibición podría no solo restringir la facultad reguladora del legislador sino el actuar mismo de la administración pública en general, pues una vez el Estado ha asumido directamente la prestación de un servicio tiene la obligación de mantener su prestación en condiciones de calidad para los usuarios del mismo. Lo anterior podría poner en apuros a los gobiernos que velan por el mantenimiento de la calidad en el servicio en momentos de emergencia social o dificultad económica.

Es entonces el derecho a la salud un derecho programático de desarrollo progresivo y no estático como se quiso dar a entender en un inicio ${ }^{33}$. Es decir que la protección no es incompatible con el principio de la progresividad de los derechos económicos y sociales: "que a medida que pasan los años si las autoridades encargadas no han tomado medidas efectivas que aseguren avances en la realización de las prestaciones protegidas por los derechos constitucionales, gradualmente van incurriendo en un incumplimiento [...] que una prestación amparada por un derecho sea de carácter programático no quiere decir que no sea exigible o que enteramente pueda incumplirse". Es así como la Corte da a entender que si hay incumplimiento por las autoridades en el desarrollo de derechos programáticos, estos podrían, en ciertos casos, ser exigibles inmediatamente a través de los mecanismos legales y constitucionales dirigidos para tal fin.

Por tal motivo la doctrina ${ }^{34}$ ha tratado de establecer algunos límites, y ha considerado que de forma general el derecho a la salud no puede ser considerado como un derecho fundamental. Esto tiene que ver no solamente con la transformación constitucional de la naturaleza de un derecho sino por las graves implicaciones que esto genera en materia estructural y financiera para el gobierno

33 Corte Constitucional. Sentencia T-595 de 2002.

34 M. Hernández, El derecho a la salud en Colombia: obstáculos estructurales para su realización, en: La salud está grave, una visión desde los derechos humanos, Provea, Bogotá (2000). 
de turno. Esto significa que el derecho a la salud, al ser protegido directamente por vía de tutela, en todos los casos pondría en jaque al Estado, ya que la protección de estos derechos está ligada íntimamente al desarrollo político, económico y social de un país, y que dentro del ordenamiento jurídico existen diferentes entes, tanto administrativos como judiciales, para velar por su protección; el problema sería entonces más estructural que conceptual.

La justicia constitucional parece aceptar parcialmente la anterior posición cuando precisa que los derechos prestacionales pueden transformarse en derechos subjetivos solamente en ciertos casos: "las condición meramente programática de los derechos económicos, sociales y culturales tiende a transmutarse en un derecho subjetivo, en la medida que se creen los elementos que le permitan a la persona exigir del Estado la obligación de ejecutar una prestación determinada, consolidándose entonces el deber asistencial en una realidad concreta a favor de un sujeto especifico" 35 .

Por consiguiente, en la medida en que el Estado se desarrolla socialmente, se va dando aplicación al principio de progresividad de los derechos económicos y sociales, y crea una estructura tanto administrativa como financiera para su especial protección, estos van convirtiéndose en derechos subjetivos pudiendo ser protegidos por el juez constitucional mediante las garantías consagradas en la Carta magna ${ }^{36}$.

Se debe tener en cuenta que el derecho a la salud se presenta como fundamental y autónomo frente a sujetos de especial protección. Es decir, que nuestra Carta Política, con fundamento en el principio a la igualdad en sentido material, propia del Estado social de derecho, identifica a sujetos que merecen especial protección, situación que la jurisprudencia ha denominado como "protección del derecho a la salud reforzada"37, y que sus demandas de protección las trata, no como derecho conexo, sino como derecho autónomo, cuando este tiene relación directa con los derechos de los adultos mayores, los discapacitados, los menores de edad, las personas con enfermedades catastróficas, las mujeres embarazadas, entre otras ${ }^{38}$.

35 Corte Constitucional. Sentencia T-304 de 1998.

36 A. Román, Análisis de la jurisprudencia constitucional sobre el sistema general en seguridad en salud, tendencias, problemática y perspectivas, Vniversitas, No. 97 (1997).

37 Corte Constitucional. Sentencia T-1081 de 2003.

38 Corte Constitucional. Sentencia, T-1185 de 2005. 
A pesar de toda esta protección especial, la justiciabilidad del derecho a la salud sigue siendo frágil e incierta.

\section{LA FRAGILIDAD DE JUSTICIABILIDAD DEL DERECHO A LA SALUD EN COLOMBIA}

Como lo vienen denunciando cada vez más los organismos de control, el balance realizado en materia de tutela hasta el 2012 sigue siendo preocupante en materia de salud. Del total de las tutelas interpuestas en el país en 2010: 403.380, con aumento considerable del $8,83 \%$, representan en salud 94.502 tutelas, que equivale al $23,4 \%$. Esto es considerable si se tiene en cuenta que desde la Sentencia T-760 de 2008, considerada una verdadera política pública en la materia, se han realizado correctivos y se han implementado nuevas legislaciones, pero la situación sigue igual de preocupante y la solución parece no existir. Una nueva reforma se anuncia y las expectativas no son las mejores en materia de protección.

\section{A. Discusiones acerca de la justiciablidad del derecho a la salud}

Si bien es cierto que en nuestros días ya nadie acata seriamente el papel que juega el juez de tutela en la protección de los derechos fundamentales ${ }^{39}$ para un adecuado funcionamiento de las instituciones de un Estado social de derecho ${ }^{40}$, también es cierto que la intervención del juez constitucional en el derecho a la salud, como uno de los derechos sociales fundamentales que ingresaron a la lista por medio de la interpretación conexa con derecho a la vida ${ }^{41}$, ha generado mucha polémica en nuestro país.

Lo anterior porque dicha protección ha hecho entrar juego dos elementos descartados por el juez constitucional colombiano: la sustentabilidad financiera y las reglas actuales del mercado. Este desconocimiento, además, ha generado un impacto considerable, no solamente en el sistema judicial, el cual se encuentra colapsa-

39 John J. Rawls, La teoría de la justicia trad. María Dolores González, $2^{\circ}$ ed., Fondo de Cultura Económica, México (1995)

40 R. Alexy, Teoría de los derechos fundamentales, trad. Ernesto Garzón Valdés, Centro de Estudios constitucionales, Madrid (1997)

41 Corte Constitucional. Sentencia T-406 de 1992. 
do por el excesivo proteccionismo, sino también por el problema económico que le ha generado al gobierno el tener que afrontar un cubrimiento de servicios sociales no previstos en el presupuesto anual. Esto conlleva a un problema de funcionalidad estatal, en donde la justicia constitucional parece no ponerse de acuerdo con el gobierno al momento de garantizar los derechos de las personas. Da la impresión de que la justicia constitucional, al momento de proteger el derecho a la salud, no tiene en cuenta el contexto en el que se aplica el texto constitucional: un sistema imperfecto, débil y subdesarrollado; pero tampoco la disponibilidad de recursos al momento de garantizar la protección de los derechos sociales ni la preparación, ocupación y congestión de los jueces al momento de tratar volúmenes insospechados de tutelas.

Dentro de este desbalance, se ha tratado de fundamentar la justicialidad de los derechos sociales ${ }^{42}$ por medio de un tratamiento como derechos subjetivos ${ }^{43}$. Es decir, que dichos derechos tendrían el poder legal para que un sujeto por medio de una norma legal exija de otros un hacer, un permitir o un omitir algo para garantizar el libre ejercicio de sus intereses. Con esto se sostiene que la justicialidad materializada por los jueces de tutela es otro elemento importante para darles dicha categoría.

Con lo anterior ${ }^{44}$, el tratamiento fundamental de estos derechos no es sino un ejemplo del constitucionalismo como nuevo paradigma del derecho. El nuevo constitucionalismo resulta de la positivización de los derechos fundamentales como límites y vínculos sustanciales a la legislación positiva, lo que implica una segunda revolución en la naturaleza del derecho que se traduce en una alteración interna del paradigma positivista clásico. Si la primera revolución se expresó mediante la afirmación de la omnipresencia del legislador con la expedición formal de normas, la segunda revolución consiste en la afirmación del principio de legalidad sustancial. Esta es la tendencia que ha seguido la Corte Constitucional durante veinte años de protección del derecho a la salud como derecho fundamental.

42 R. Arango, El concepto de derechos sociales fundamentales, Legis - Universidad Nacional de Colombia, Bogotá (2005).

43 A. Estrada, Teoría de los derechos públicos subjetivos en la obra de Georg Jellinek, Universidad Externado de Colombia, Col. Temas de derecho público, No. 48, Bogotá (1997).

44 L. Ferrajoli, Los fundamentos de los derechos fundamentales, Trotta, Barcelona (2007). 
Sin embargo, se sostiene desde hace algún tiempo ${ }^{45}$ que dicha categoría no puede quedar sujeta a la libre interpretación judicial; esto tendría un problema de graduación y podría incurrir en desigualdades más grandes que las que pretende solucionar la protección constitucional ${ }^{46}$. Además, por ser derechos prestacionales, programáticos y de garantía progresiva, antes de reconocerlos como fundamentales debería tenerse en cuenta la competencia del legislador dentro del Estado, las condiciones económicas del gobierno, el funcionamiento del mercado y el sistema jurídico que lo protege ${ }^{47}$.

Si bien es cierto que estos derechos han sido reconocidos por diferentes organismos internacionales ${ }^{48}$, esto no sería suficiente para garantizar su eficacia de forma inmediata ${ }^{49}$. La vigencia de los derechos sociales son obligaciones estatales de prestación que dependen de factores extrajurídicos tales como las condiciones económicas y sociales del Estado ${ }^{50}$. Por consecuencia, de nada vale garantizar formalmente la exigibilidad de dichos derechos si el Estado no cuenta con las condiciones económicas para cumplir las decisiones judiciales ${ }^{51}$.

En esta controversia ${ }^{52}$ existe una posición que defiende los derechos de las personas frente a las necesidades del Estado, por los beneficios sociales y la justicia constitucional que predominan en un Estado social de derecho. Otra, por el contrario, considera que la garantía de este tipo de derechos fundamentales tiene límites y por mucho que se desee ampliarlos, esto se convierte finalmente en una utopía social ${ }^{53}$ que ningún Estado ha podido realizar hasta el momento.

Lo cierto es que para la Corte Constitucional no hay justificación válida para que el derecho a la salud, como derecho social, siga sien-

45 H. Kelsen, Teoría pura del derecho, trad. Roberto Vernengo, Universidad Autónoma Nacional de México, México D.F. (1986).

46 R. Alexy, El concepto y la validez del derecho, Gedisa, Barcelona, 87 (1997).

47 C. Molina, Corte Constitucional y Estado social de derecho, Col. Memorias Jurídicas, No. 4, Universidad de Medellín (2007).

48 oms - Unicef, Declaración de Alma Alta, en: oms - Unicef, Salud para todos en el siglo XXI: Ginebra.

49 R. García Macho. Las aporías de los derechos fundamentales sociales y el derecho a una vivienda, Instituto de Administración local, Madrid (1999).

50 S. Clavijo, Fallos y fallas de las Corte Constitucional, Alfaomega, Cambio, Bogotá (2001).

51 C. Hesse, Significado de los derechos fundamentales, en: vVAA, Manual de derecho constitucional, Instituto de Administración Pública, Marcial Pons, Madrid (1996).

52 C. Molina, Controversias constitucionales, Universidad del Rosario, Bogotá (2009).

53 S. Clavijo, Descubriendo la nueva Corte Constitucional, Alfaomega, Cambio, Bogotá (2004). 
do tratado dentro de la categoría secundaria de derechos abstractos, contraria a la categoría principal y específica de derechos civiles y políticos. Lo que frecuentemente ha justificado para aquellos una ausencia de protección jurisdiccional y que en nuestros días, a causa del activismo judicial, aqueja una sobredimensión de protección ${ }^{54}$.

\section{B. Límites a la justiciabilidad del derecho a la salud}

Frente a los graves problemas que afecta la protección del derecho a la salud, la jurisprudencia que lo garantiza encuentra varios límites propios del sistema constitucional que los reconoce ${ }^{55}$.

Aparte del grave problema de exceso de demanda, el cual difícilmente puede ser absorbido por el sistema judicial actual, y de la grave crisis financiera y presupuestal del gobierno, la cual escasamente puede atender dado el exceso de tutelas en salud, se presenta otro grave problema para el sistema de protección: el vacío democrático ${ }^{56}$.

En efecto, en primer lugar, la justiciabildad del derecho a la salud como derecho social ha generado un grave enfrentamiento entre los poderes del Estado por supuesta usurpación o extralimitación de competencias ${ }^{57}$. Las denuncias no son pocas en contra de un juez co-legislador ${ }^{58}$ que parece desconocer el principio de separación de poderes y la jerarquía normativa ${ }^{59}$. En segundo lugar, se le acusa al juez constitucional, en su activismo judicial en salud ${ }^{60}$, de reducir la discusión democrática, tomando en poco tiempo, y por grupos de tres magistrados, decisiones que en el Congreso deben tomar meses de discusión y de debate democrático de amplias mayorías democráticas y representativas de la sociedad, en materia de la política

54 J. Núñez, Justicia social por vía judicial: manifiesto por una justicia constitucional responsable, Legis, Bogotá (2005).

55 M. Correa Henao, La limitación de los derechos fundamentales, Universidad Externado de Colombia, Col. Temas de derecho público, No. 71, Bogotá (2003).

56 C. Molina, Protección de derechos sociales y economía, Revista de Derecho Económico, No. 6 , Universidad Javeriana, Bogotá, 552 (2011).

57 G. Caballero Sierra, Corte Constitucional y legislador: contrarios o complementarios, Ediciones Ibáñez, Col. Estudios Breves, Bogotá (2002).

58 S. Morelli Rico, La Corte Constitucional: ¿un legislador complementario?, Universidad Externado de Colombia, Col. Temas de derecho público, No. 45 Bogotá.

59 D. de Bechillon, Hiérarchie des normes et hiérarchie des fonctions normatives de l'Etat, Economica, Paris (1996).

60 C. Molina, Reflexiones acerca del activismo judicial en materia de derechos sociales en Colombia: el caso del derecho a la salud, Revista Elementos de Juicio, No. 13, 91-113 (2010). 
pública en salud, lo que arriesga considerablemente a judicializar la política en Colombia ${ }^{61}$.

Esto parece haber sucedido en el actual gobierno, que propone ahora una nueva reforma al sistema. El nuevo documento recoge algunos temas de la ponencia para primer debate del Proyecto de Ley 048 de 2012 - Senado, presentado por el H. Senador Armando Benedetti, el cual surge de la concertación con la Gran Junta Médica, que impulsó decididamente el enfoque del Proyecto de Ley 209 de 2013 Senado - 267 de 2013 Cámara, presentado el pasado 19 de marzo del 2013. Dichas propuestas se reúnen simultáneamente con el Proyecto de Ley 210 de 2013 de iniciativa gubernamental, a través del cual se desarrollará una redefinición del Sistema General de Seguridad Social en Salud - SGSSS.

La propuesta tiene en cuenta la Observación General 14 de 2000 del Comité de Derechos Económicos y Sociales, que señala que "la salud es un derecho humano fundamental e indispensable en el ejercicio de los demás derechos humanos". Asimismo, en las sentencias C-463 y T-760 de 2008, con lo que el legislador puede expedir una norma con el carácter estatutario, dentro de los límites y linderos propios de una disposición de tal naturaleza.

Lo que pretende la actual reforma a la salud es la creación de un sistema autónomo que garantice una mayor cobertura, con una sólida coherencia en su estructura y una mayor eficiencia en la asignación de los recursos, bajo un esquema compartido de triple hélice EMS (Estado-Empresa-Sociedad), que enmalla el sistema bajo parámetros internacionales que garantizan universalidad, libertad de elección, solidaridad y equidad ${ }^{62}$.

Con este proyecto de ley, los colombianos podrían acceder a un servicio de salud oportuno, transparente y de calidad. El proyecto plantea un nuevo modelo de salud más eficiente y que asegure completamente un servicio sin intermediación financiera. El proyecto incluye cuatro grandes fórmulas:

61 R. Uprimny-Yepes, La justice au cœur du politique: potentialités et risques d'une judiciarisation en Colombie, en Commaille J. et Kalubzynski M., La fonction politique de la justice, Editorial La Découverte, Paris, 229-250.

62 A. Gaviria \& M. Cárdenas. Respuestas del Gobierno y el Ministerio de Salud a las preguntas sobre reforma a la salud, Presidencia de la República, Urna de Cristal, Bogotá (2013). Disponible en línea: http://www.urnadecristal.gov.co/gestion-gobierno/respuestas-reforma-salud-colombia 
a. Salud-Mía: un fondo único que administra los recursos del sistema de salud.

b. Mi-Plan: un plan de beneficios único que incluya todas las patologías.

c. Los gestores de servicios de salud: que acompañarán a los ciudadanos por su paso por el sistema de salud, que respondan por la salud de sus afiliados.

d. Un sistema de prestación de servicios organizado por áreas de gestión sanitaria, con redes de servicios cercanas a los ciudadanos.

Dicho esquema es ambicioso y organizaría de forma definitiva el nuevo sistema de salud universal en Colombia, pero deja grandes inquietudes. En primer lugar, hace depender en cierta medida la salud de la estabilidad laboral y de la economía de todo un país. De esta forma, desde su puesta en marcha, la cobertura del servicio ha fluctuado, en calidad y cobertura, de acuerdo con la evolución del mercado y su capacidad para generar empleo. Por ello, mucho se piensa ahora sobre el balance de dos décadas del sistema, del cual no se han visto hasta ahora todas sus bondades.

En este sentido ${ }^{63}$, se ha podido detectar desde hace algún tiempo que algunas fallas son visibles en un sistema montado bajo estos esquemas: mala información de morbilidad, mala distribución de ingresos entre las empresas prestadoras del servicio, mala actualización de las bases de datos de los afiliados, tanto en el régimen contributivo como en el subsidiado y poca ampliación en cobertura y vigilancia y control de parte del Estado. Esto se ha mejorado, pero su falta universal de coherencia y eficacia sigue generando un grave problema para la competitividad, calidad y transparencia en la oferta del servicio. Pareciera que en estos 20 años de privatización del sistema, el servicio de salud se hubiera dedicado más a solicitar recursos, pidiendo el aumento del UPC, que a mejorar la prestación del servicio. Estos estudios revelan que muchos de los prestadores del servicio no llevan estadísticas de mortalidad ni de tratamiento de enfermedades, por el simple hecho de que desconocían dicha función. Muchos de los prestadores del servicio, por el afán de no

63 M. Martínez, F. Robayo \& O. Valencia, ¿Por qué no se logra la cobertura universal de la seguridad social en salud?, Fundación para la investigación y desarrollo de la salud y la seguridad social Fedesalud, Bogotá, 6 (2002). 
incurrir en altos gastos, niegan sistemáticamente los servicios de salud, para que las tutelas los obliguen a pagar y así ellos puedan hacer el recobro al Fosyga (Fondo de Solidaridad y de Garantías).

En segundo lugar, el problema del volumen de las tutelas es otro elemento que no parece quedar resuelto en el proyecto, si bien es cierto que las reformas realizadas hasta ahora no han permitido que el nuevo sistema muestre todas sus bondades ${ }^{64}$. Igualmente es incierto el futuro de este mecanismo constitucional de protección constitucional de uno de los derechos más vulnerados en el país. El proyecto de ley no desconoce el derecho a la tutela, argumentando que solamente amplía el plan de beneficios, de manera que los colombianos no tendrían que acudir ni a ese mecanismo, ni a los comités técnico-científicos, para que les autoricen medicamentos y servicios médicos.

Sin embargo, sigue sin atacarse de fondo el problema actual, que más del 65\% de las tutelas interpuestas anualmente se refieren al Sistema de Seguridad Social, de las cuales, 70\% aproximadamente tienen que ver con salud y de ellas un gran número es sobre derechos que ya tienen reconocidos los usuarios. Las estadísticas más recientes ${ }^{65}$ tienen establecido que en el país se interponen más de 3.000 tutelas cada mes, es decir unas 172 tutelas diarias, lo que se convierte en 22 tutelas por hora; es decir 2,7 tutelas por minuto en materia de salud (4 o 5 en todas las áreas). Las tutelas en salud se conceden en un $60 \%$, que en buen número afectan al Fondo de Solidaridad y Garantía (Fosyga) del Ministerio de Protección Social. De estas, cerca de un 30\% se interponen para reclamar servicios de salud cubiertos por el POS, $60 \%$ por fuera del pos y $10 \%$ tienen que ver con demandas que estaban por fuera de las cotizaciones mínimas exigidas para la atención.

Lo que representa, en ciertos casos, un costo considerable para el Ministerio de la Protección Social que destina, aproximadamente, unos $\$ 75$ mil millones para dicha atención, sin contar con los altos costos que representa ahora la atención de enfermedades

64 Organización Iberoamericana de Seguridad Social, Instituto de Ciencias de la Salud cesMedellín, Trascendencia e impacto socioeconómico de la acción de tutela en el Sistema de Seguridad Social Colombiano, Universidad Javeriana, Bogotá (2005).

65 C. Molina, La tolerancia en la jurisprudencia de la Corte Constitucional: el caso del derecho a la salud, en: Tolerancia y derecho, Sello Editorial Universidad de Medellín, 265-282. ISBN. 978-958-8692-41-8 (2012). 
no convencionales como el SIDA, el alcoholismo, el consumo de drogas, los diferentes tipos de intervenciones quirúrgicas, algunas efectuadas en el exterior y otras muy publicitadas como la del Bypass gástrico. En el 80\% de los casos los jueces les dan la razón a los ciudadanos, y de ese porcentaje el $70 \%$ es confirmado por la Corte Constitucional. Dicha tendencia se mantiene, con un ligero aumento en ciertos años. El exceso sigue aumentando sin control. En el 2010, el Ministerio de la Protección Social pagó por recobros 2,3 billones y en el 2012 1,8 billones por comités técnico-científicos y por tutelas no pos. Esto es alarmante si se tiene en cuenta que del presupuesto nacional en salud, 45.518 .424 billones (8,30\% del PIB), el Fosyga maneja 22.984 .485 billones de pesos, lo que equivale al $4,85 \%$ del PIB.

Este crecimiento desmesurado de la demanda de protección y los patrones de desfase presupuestal constante ${ }^{66}$ han llevado a proponer desde tiempo atrás modificaciones a las normas que regulan la tutela, con la intención de regular el empleo de este mecanismo en aquellos sectores que se han considerado como sensibles desde el punto de vista presupuesta ${ }^{67}$. Sin embargo, este tipo de propuestas no han sido atendidas en la actual discusión parlamentaria sobre la reforma al sistema y dejan por fuera el análisis de los factores institucionales que inciden o propician el empleo de la tutela como mecanismo para solucionar problemas institucionales, tanto de tipo normativo como organizacional y de control ${ }^{68}$.

Por último, el actual proyecto en discusión trae unos límites, que han sido siempre considerados como los obstáculos a la garantía de proteccionismo en salud: el reconocimiento expreso de la naturaleza económica del derecho. Es así como el artículo 4 del nuevo proyecto, que trata los elementos y principios del derecho fundamental a la salud, reconoce que el derecho fundamental a la salud incluye los siguientes elementos esenciales e interrelacionados:

66 Defensoría del Pueblo, La tutela y el derecho a la salud causas de la tutela en salud, Defensoría del Pueblo, Bogotá (2003).

67 Defensoría del Pueblo, El derecho a la salud en la Constitución, la Jurisprudencia y los mecanismos internacionales, Bogotá (2004).

68 "Bajo presión, el Congreso define futuro de 26 billones en salud", El Colombiano, Medellín, 2 (2013) 
g) Progresividad del derecho. El Estado promoverá la correspondiente ampliación gradual y continua del acceso a los servicios y tecnologías de salud, la mejora en su prestación, la ampliación de capacidad instalada del sistema de salud y el mejoramiento del talento humano, así como la reducción gradual y continua de barreras culturales, económicas, geográficas, administrativas y tecnológicas que impidan el goce efectivo del derecho fundamental a la salud.

i) Sostenibilidad. El Estado dispondrá, por los medios que la ley estime apropiados, los recursos necesarios y suficientes para asegurar progresivamente el goce efectivo del derecho fundamental a la salud.

Deja entonces en manos del gobierno el financiamiento gradual y progresivo del servicio. Dichas limitantes se acuerdan con lo establecido en el Proyecto de Ley 06 aprobado en Comisión Séptima del Senado de noviembre del 2012, que propone un manual de tarifas mínimas en salud. Pero lo más preocupante del tema ${ }^{69}$ es la aparente voluntad del gobierno de hacer prevalecer el equilibrio fiscal sobre la protección inmediata del derecho a la salud. Se ha discutido en este sentido un mecanismo incidental que le permita al Ministerio Público o al Ministro de Hacienda frenar una decisión de tutela cuando esta atente contra el equilibrio presupuestal de financiamiento del sistema.

Todo esto confirma la voluntad del gobierno de frenar el activismo judicial en materia de tutela e impedir al máximo que los jueces le establezcan al gobierno la política pública en materia de salud. Veremos en qué para este nuevo enfrentamiento institucional o "choque de trenes" que se avecina. 


\section{CONCLUSIóN}

En el grupo de los derechos sociales, económicos y culturales, el avance normativo colombiano en materia de salud ha sido de los más destacados en el mundo. Los últimos cincuenta años han sido de evolución en lo que tiene que ver con el acceso al servicio público de atención en salud.

En estricta definición jurídica, la naturaleza del servicio de salud es un derecho esencial, mas no fundamental, no solamente por no ser catalogado como individual dentro de la Carta Constitucional de 1991, sino porque histórica y estructuralmente siempre ha sido considerado como un derecho prestacional a cargo del Estado. Esto tiene una buena explicación en la teoría del servicio público, dirigido y reglamentado por el Estado, y no impuesto a este por encima de principios supraconstitucionales. Con esto, es el legislador colombiano el llamado a regular la prestación y protección de todo el Sistema de Seguridad Social, de acuerdo a la evolución del Estado y de los recursos con que cuenta.

La Corte Constitucional ha obviado esta naturaleza y ha reconocido en ciertos casos la prestación de salud como un derecho subjetivo, lo que ha generado problemas de financiación y desorden en la estructura del Sistema de Seguridad Social. Su jurisprudencia garantista ha ordenado al gobierno de turno cubrir muchos servicios que en principio no deberían ser cubiertos por medio de una acción de tutela, lo que ha generado grandes expectativas en la colectividad más desfavorecida. El precio de tanto proteccionismo ha sido grande para la teoría de fuentes del derecho, para la seguridad y estabilidad normativa y para la uniformidad del sistema de protección, el cual parece colapsado.

No obstante el desarrollo importante que ha tenido la protección del derecho a la salud en el mundo y en Colombia en los últimos cincuenta años, y a pesar de todos estos esfuerzos estructurales y avances jurisprudenciales, el Estado no ha podido consolidar las instituciones que, a través de su papel regulador, de dirección y ejecución, garanticen la calidad en los servicios de salud.

El Estado deberá asumir la redefinición y redirección del sistema, para que los actores de los mismos apunten al cumplimiento de políticas públicas de salud en el marco de la promoción y preven- 
ción y no solo en el manejo de la morbilidad, teniendo en cuenta la importancia social y económica que reviste una sociedad. El actual sistema requiere una revisión profunda para seguir evitando el desmonte de un sistema que ha mostrado un cierto progreso.

En síntesis, el sistema de salud hay que revisarlo a profundidad y la protección judicial regularla, pero sin renunciar a los grandes avances que se han llegado. De todas formas, si no hay un acuerdo entre los poderes del Estado, la crisis sería inminente y quien pagaría finalmente las consecuencias es la población más vulnerable. 


\section{BIBLIOGRAFÍA}

\section{Libros}

A. Estrada, Teoría de los derechos públicos subjetivos en la obra de Georg Jellinek, Universidad Externado de Colombia, Col. Temas de derecho público, No. 48, Bogotá (1997).

A. Rodríguez Castillo, La garantía de la salud como derecho integral en Colombia, Diálogo pendiente, Ed Planeta Paz, Bogotá (2005).

C. Molina, Controversias constitucionales, Universidad del Rosario, Bogotá (2009).

C. Molina, Corte Constitucional y Estado social de derecho, Col. Memorias Jurídicas, No. 4, Universidad de Medellín (2007).

C. Molina. El derecho al aborto: segunda parte, la despenalización parcial, Universidad de Medellín. ISBN. 978-958-8348-97-1.

D. de Bechillon, Hiérarchie des normes et hiérarchie des fonctions normatives de l'Etat, Economica, París (1996).

Defensoría del Pueblo, El derecho a la salud en la Constitución, la Jurisprudencia y los mecanismos internacionales, Defensoría del Pueblo, Bogotá (2004).

, La tutela y el derecho a la salud causas de la tutela en salud, Defensoría del Pueblo, Bogotá (2003).

G. Caballero Sierra, Corte Constitucional y legislador: contrarios o complementarios, Ediciones Ibáñez, Col. Estudios Breves, Bogotá (2002).

H. Hart, El concepto de derecho, Abeledo Perrot, Buenos Aires (1990).

H. Heller. Teoría del Estado, Fondo de Cultura Económica, México D.F. (1983).

H. Kelsen, Teoría pura del derecho, trad. Roberto Vernengo, Universidad Autónoma Nacional de México, México D.F. (1986).

J. Fernández, El servicio público de la sanidad: el marco constitucional, Civitas, Madrid, 82 (1984).

J. Núñez, Justicia social por vía judicial: manifiesto por una justicia constitucional responsable, Legis, Bogotá (2005).

J. Pérez Royo, El Estado social de Derecho, en: Enciclopedia Jurídica Básica, Vol. III, Civitas, Madrid (1989).

John J. Rawls, La teoría de la justicia, trad. María Dolores González, 2ª ed., Fondo de Cultura Económica, México (1995).

L. Ferrajoli, Los fundamentos de los derechos fundamentales, Trotta, Barcelona (2007).

M. Arbeláez Rudas, Derecho a la salud en Colombia, el acceso a los servicios del sistema general de seguridad social en salud, IDEP - CINEP - Colciencias, Bogotá (2006).

M. Correa Henao, La limitación de los derechos fundamentales, Universidad Externado de Colombia, Col. Temas de derecho público, No. 71, Bogotá (2003).

M. Hernández, El derecho a la salud en Colombia: obstáculos estructurales para su 
realización, en: La salud está grave, una visión desde los derechos humanos, Provea, Bogotá (2000).

M. Martínez, F. Robayo \& O. Valencia, ¿Por qué no se logra la cobertura universal de la seguridad social en salud? Fundación para la investigación y desarrollo de la salud y la seguridad social Fedesalud, Bogotá, 6 (2002).

M. Mazziotti, Diritti sociali, Milán, D.T.XII (1964).

N. Paredes, El Derecho a la salud: su situación en Colombia, CINEP - GTZ, Bogotá (2003).

N. Paredes, El Derecho a la salud: su situación en Colombia, CINEP - GTZ, Bogotá (2003).

O. Cetina Vargas, Derecho Integral de Seguridad Social, Universidad Externado de Colombia, Bogotá (1986).

O. Correas, Acerca de los derechos humanos, Ediciones Coyoacán, México (2003).

O. Cortés Hernández, Derecho a la Seguridad Social, 2a ed., Librería Ediciones del Profesional Ltda., Bogotá, 3 (2006).

Organización Iberoamericana de Seguridad Social, Instituto de Ciencias de la Salud CES-Medellín, Trascendencia e Impacto Socioeconómico de la Acción de Tutela en el Sistema de Seguridad Social Colombiano, Universidad Javeriana, Bogotá (2005).

Procuraduría General de la Nación, Informe en Salud, Bogotá (2008).

R. Alexy, El concepto y la validez del derecho, Gedisa, Barcelona (1997).

, Teoría de los derechos fundamentales, trad. Ernesto Garzón Valdés, Centro de Estudios constitucionales, Madrid (1997).

R. Arango, El concepto de derechos sociales fundamentales, Legis - Universidad Nacional de Colombia, Bogotá (2005).

R. García Macho, Las aporías de los derechos fundamentales sociales y el derecho a una vivienda, Instituto de Administración local, Madrid (1999).

S. Clavijo, Descubriendo la nueva Corte Constitucional, Alfaomega, Cambio, Bogotá (2004). (2001).

S. Morelli Rico, La Corte Constitucional: ¿un legislador complementario?, Universidad Externado de Colombia, Col. Temas de derecho público, No. 45 Bogotá.

\section{Contribuciones en obras colectivas}

C. Hesse, Significado de los derechos fundamentales, en: vvaA, Manual de derecho constitucional, Instituto de Administración Pública, Marcial Pons, Madrid (1996).

C. Molina, La tolerancia en la jurisprudencia de la Corte Constitucional: el caso del derecho a la salud, en: Tolerancia y derecho, Sello editorial Universidad de Medellín, 265-282. ISBN. 978-958-8692-41-8 (2012).

M. Restrepo, Elementos para una doctrina del Derecho a la salud en Colombia, en: El 
ejercicio del derecho a la salud en Colombia, Defensoría del Pueblo, Bogotá (2002).

OMs - Unicef, Declaración de Alma Alta, en: oms - Unicef, Salud para todos en el siglo $X X I$ : Ginebra.

OMS - Unicef, Salud para todos en el siglo XXI, en: Declaración de Alma Alta, Ginebra (1999).

R. Uprimny-Yepes, La justice au cœur du politique: potentialités et risques d'une judiciarisation en Colombie, en: J. Commaille y M. Kalubzynski, La fonction politique de la justice, Editorial La Découverte, Paris, 229-250.

W. Abendroth, El Estado de derecho democrático y social, en: vvvaA, El Estado social, Centro de Estudios Constitucionales, Madrid (1986).

\section{Revistas}

A. Román, Análisis de la Jurisprudencia Constitucional sobre el sistema general en seguridad en salud, tendencias, problemática y perspectivas, Vniversitas, No. 97 (1997).

C. Herrera, Sur le statut des droits sociaux. La constitutionnalisation du social, 16 RUDH, No. 1-4, 33 (2004).

C. Molina, Protección de Derechos sociales y economía, Revista de Derecho Económico, No. 6, Universidad Javeriana, Bogotá, 552 (2011).

, Reflexiones acerca del activismo judicial en materia de derechos sociales en Colombia: el caso del derecho a la salud, Revista Elementos de Juicio, No. 13, 91-113 (2010).

Contraloría General de la República, El sistema nacional de salud diez años después de la ley 100, Revista Economía Colombiana, No. 303 (2003).

E. Cobrero, Reflexión general sobre la eficacia normativa de los principios constitucionales rectores de la política social y económica del Estado, Revista Vasca de Administración Pública, No. 19, 221 (1987).

N. Ardón \& A. Centeno, Voces críticas desde la academia sobre La emergencia social en salud. Cuadernos del doctorado, No.13, Universidad Nacional de Colombia, Bogotá (2006). Disponible en http://web.saludsantander.gov.co/index. php?option=com_docman\&task=doc_download\&gid=263\&Itemid=7

\section{Artículos de prensa}

Bajo presión, el Congreso define futuro de 26 billones en salud, El Colombiano, Medellín, 2 (2013).

F. Londoño Hoyos, (Extraña Paradoja), El Tiempo, 11 de febrero del 2011.

R. Arango, Adiós a las tutelas, El Espectador, 6 de junio del 2013, 30, Bogotá (2013). 


\section{Documentos en Internet}

A. Gaviria \& M. Cárdenas, Respuestas del Gobierno y el Ministerio de Salud a las preguntas sobre reforma a la salud, Presidencia de la República, Urna de Cristal, Bogotá (2013). Disponible en línea: http://www.urnadecristal.gov.co/gestiongobierno/respuestas-reforma-salud-colombia

\section{Jurisprudencia colombiana}

Corte Constitucional. Sentencia T-406 de 1992.

Corte Constitucional. Sentencia T-406 de 1993. M.P. Alejandro Martínez Caballero.

Corte Constitucional. Sentencia T-447 de 1994. M.P. Vladimiro Naranjo.

Corte Constitucional. Sentencia T-042 de 1995. M.P. Carlos Gaviria Díaz.

Corte Constitucional. Sentencia T-304 de 1998.

Corte Constitucional. Sentencia SU-508 de 2001.

Corte Constitucional. Sentencia T-595 de 2002.

Corte Constitucional. Sentencia T-1081 de 2003.

Corte Constitucional. Sentencia T-1185 de 2005.

Corte Constitucional. Sentencia T-760 de 2008. M.P. Manuel José Cepeda Espinosa.

\section{Jurisprudencia internacional}

Tribunal constitucional español. Autos ATC 519 y 564 (1984). 